\title{
The presence and spectrum of bacteria colonising mobile phones of staff and caregivers in high disease burden paediatric and neonatal wards in an urban teaching hospital in Durban, South Africa
}

\author{
Raziya Bobat ${ }^{a *}$, Moherndran Archaryb, Melissa Lawlerb, Sajeeda Mawlanac, Kimesh L Naidoo ${ }^{b}$, Sandra Maphumulo and \\ Yacoob Coovadia ${ }^{\mathrm{d}}$ \\ ${ }^{a}$ Department of Paediatrics and Child Health, Nelson R Mandela School of Medicine, University of KwaZulu-Natal, Durban, South Africa \\ ${ }^{b}$ King Edward VIII Hospital, Durban, South Africa \\ 'Enhancing Care Foundation, Durban, South Africa \\ ${ }^{d}$ Department of Microbiology, Nelson R Mandela School of Medicine, University of KwaZulu-Natal and National Health Laboratory Services, \\ KawZulu-Natal, South Africa \\ *Corresponding author, email: bobat@ukzn.ac.za
}

\begin{abstract}
Background: Hospital acquired infections are on the increase worldwide. A possible source for transmission is the presence of microorganisms on mobile phones which are carried by increasing numbers of medical and nursing staff, students, and caregivers.

Methods: A cross-sectional study was performed. Samples were obtained from medical and nursing staff, students, and caregivers from the paediatric wards ( 85 beds), and the neonatal unit ( 40 beds) at King Edward VIII Hospital (KEH), Durban. Mobile phones of participants were sampled, with sterile swabs, without prior warning. The swabs were transported promptly to the laboratory and cultured onto colistin, nalidixic acid agar and MacConkey agar plates. All positive cultures were identified using standard laboratory tests.

Results: Of the 100 mobile phones sampled, 30 were contaminated with bacteria. Gram-positive microorganisms were more frequently cultured than Gram-negative microorganisms (29 vs. 7). Significantly more caregivers had contaminated phones $(54.17 \%$ vs. $22.37 \%, p$ value $=0.003)$. Caregivers' phones were predominantly contaminated with Staphylococcus spp. (41.67\%). More phones in the general wards (37.5\%) and nursery (32.5\%) were contaminated compared to the gastroenteritis ward (10\%). Conclusions: Our results indicate that one third of the mobile phones carried in the paediatric wards are contaminated. Caregivers had the highest likelihood of carrying mobile phones that were contaminated, with the lowest rate among the nursing staff. Caregivers' phones were also more likely to have multiple microorganisms. The lowest rate of contamination was found in the gastroenteritis ward, possibly due to a heightened awareness of the need for handwashing.
\end{abstract}

Keywords: contamination, hospital-acquired infections, mobile phones

\section{Introduction}

Maintaining adequate infection control practices in high disease burden ${ }^{1}$ paediatric and neonatal units is an issue of ongoing concern to the paediatric staff of any hospital's Infection Prevention and Control (IPC) department. There are many possible routes of transfer of microorganisms to patients within the wards, resulting in spread of disease, prolonged admissions, and frequent readmission. Nosocomial infection continues to be of increasing significance. According to the World Health Organisation (WHO), an average of $8.7 \%$ of hospital patients have nosocomial infections. At any time, over 1.4 million people worldwide suffer from infectious complications acquired in hospital. ${ }^{2}$

One of the possible routes of transfer is the presence of microorganisms on mobile phones which are carried by all medical staff, large numbers of nursing staff, students, and almost all caregivers. Over the past decade, mobile phones have become an essential accessory in our social and professional lives, used increasingly by healthcare personnel for communication. Mobile phones may harbour many harmful pathogens, which may serve as a reservoir for nosocomial infections. ${ }^{3,4}$ High mobile phone usage is reported in South Africans across all economic groups. The General Household
Survey (GHS) conducted in South Africa in 2013 found that $81.9 \%$ of households had access to at least one mobile cellular phone. ${ }^{5}$

Mobile phones are often touched during or after the examination of patients without handwashing and are seldom cleaned. These instruments can harbor various potential pathogens and become an exogenous source of nosocomial infections, amongst hospitalised patients. ${ }^{6,7}$ In paediatric wards, caregivers (mainly mothers) are either admitted with the child or are present at the child's bedside for most of the day. They have been noted to frequently leave mobile phones on the childrens'beds, inadvertently exposing them to a number of microorganisms contaminating the phone. Older children often use the mobile phone for playing games, and younger children frequently put the phones into their mouths [personal observation]. The potential of mobile phones to spread infection is an important component of any debate in which relaxation of restriction on mobile phone use is considered. ${ }^{8}$

\section{Aims of study}

To determine the presence of and characterise the spectrum of bacteria on mobile phones belonging to medical, nursing, allied health staff, students, and caregivers. Also, to advise on best practices based on the results obtained. 
Table 1: Bacterial growth according to staff designation

\begin{tabular}{|l|c|c|}
\hline & No growth, $\boldsymbol{n}(\%)$ & Growth, $\boldsymbol{n}(\%)$ \\
\hline Hospital Staff & $59(77.63)$ & $17(22.37)$ \\
\hline Caregiver & $11(45.83)$ & $13(54.17)$ \\
\hline TOTAL & $70(70)$ & $30(30)$ \\
\hline
\end{tabular}

*Statistical analyses were performed using STATA version 11.1 (Mac).

Table 2: Bacterial growth stratified according to ward

\begin{tabular}{|l|c|c|}
\hline & No growth, $\boldsymbol{n}(\%)$ & Growth, $\boldsymbol{n}(\%)$ \\
\hline General wards & $25(62.5)$ & $15(37.5)$ \\
\hline Nursery & $27(67.5)$ & $13(32.5)$ \\
\hline GE ward & $18(90)$ & $2(10)$ \\
\hline TOTAL & $70(70)$ & $30(30)$ \\
\hline
\end{tabular}

*Statistical analyses were performed using STATA version 11.1 (Mac).

\section{Study design}

A cross-sectional study was performed. Samples were obtained between 10:00am and 12:00am, from all the paediatric wards (85 beds), and the neonatal unit (40 beds) at King Edward VIII Hospital (KEH), Durban. KEH is a large urban teaching hospital serving a high disease burdened area with high rates of pathologies related to human immunodeficiency virus, tuberculosis and malnutrition. Permission was obtained from the Ethics committee of the University of KwaZulu-Natal and KEH Management.

After verbal consent was obtained, mobile phones of all participants were sampled without prior warning. The study was anonymous, unlinked, and non-invasive. The staff were not aware that such a survey was going to be carried out. Information that was collected included ownership of the phone (medical staff, nursing staff, allied health professional, students, or caregiver) and the type of ward from which the sample was taken.

\section{Sample collection}

Swabs were collected from 100 mobile phones of staff members, patients' caregivers, and other healthcare workers (HCW) in three paediatric wards and the neonatal nursery between 10:00am and 12:00am on a single day. Mobile phones of all participating members were swabbed on both sides using commercially available sterile swabs in Amies transport media (Clinical Sciences Diagnostics). The sterile swabs were first moistened in brain heart infusion (BHI) broth before actual swabbing of the mobile phones. The swabs were thereafter inserted into the Amies transport medium, and transported promptly to the medical microbiology laboratory at Inkosi Albert Luthuli Central Hospital (IALCH). At the laboratory, the swabs were cultured onto colistin, nalidixic acid agar (CNA) and MacConkey agar plates. The CNA agar plates were incubated at $37^{\circ} \mathrm{C}$ in a $\mathrm{CO}_{2}$ incubator whilst the MacConkey agar plates were incubated aerobically at $37^{\circ} \mathrm{C}$ for $18-24$ hours. These plates were incubated for up to 48 hours if no growth was observed at 24 hours. A semiquantitative count was performed on all positive cultures, which were subsequently identified using standard laboratory tests. ${ }^{9}$ Drug susceptibility testing was only performed on the Staphylococcus aureus isolates using the Kirby-Bauer method.

\section{Statistical analysis}

The variables were characterised into ward and staff type, and the percentages among these categorical variables were compared using the $\chi^{2}$ or Fischer's exact test, as appropriate. Statistical analyses were performed using STATA version 11.1 (Mac).

\section{Results}

A total of 100 mobile phones were sampled from 3 paediatric wards and the neonatal nursery at King Edward VIII hospital (20 from the gastroenteritis ward, 20 from each of the two general paediatric wards, and 40 from the neonatal nursery). The distribution of phones sampled was as follows: 38 from nursing staff; 24 from caregivers; 20 from doctors; and, 14 from other staff (9 medical students, 3 general orderlies, 2 occupational therapists, 2 physiotherapists and 1 speech therapist) (Table 1).

Of the 100 mobile phones sampled, 30 were contaminated with bacteria. Of these, 21 had one microorganism, 7 had two microorganisms, 1 had three microorganisms and 1 had more than three microorganisms. Gram-positive microorganisms were more frequently cultured than Gram-negative microorganisms (29 Gram-positive vs. 7 Gram-negative). The Gram-positive microorganisms cultured were Staphylococcus spp. (64\%), Staphylococcus aureus (14\%) and Micrococcus spp. (3\%). All of the S. aureus isolated were methicillin/cloxacillin sensitive. The Gramnegative microorganisms cultured were Enterobacter cloacae (8\%), Acinetobacter spp. (8\%), Pseudomonas stutzeri (3\%), Klebsiella pneumoniae (3\%) and Chryseobacterium indologenes (3\%).

Significantly more caregivers had contaminated phones (Table 1) $(54.17 \%$ vs. $22.37 \%, p$ value $=0.003)$. Caregivers' phones were predominantly contaminated with Staphylococcus spp. (41.67\%). In terms of Gram-negative microorganisms cultured, two staff member's phones harboured 1 type of Gram-negative bacteria each. In comparison, three out of 24 caregivers' phones (12.5\%) harboured 1 Gram-negative microorganism, and 2 out of 24 (8.33\%) harboured 2 or more Gram-negative microorganisms.

Analysis of bacterial contamination according to the ward from which the mobile phone was sampled (Table 2), showed that more mobile phones in the general wards (37.5\%) and nursery (32.5\%) were contaminated compared to the gastroenteritis ward (10\%). The neonatal nursery exhibited the highest diversity of contaminating microorganisms: 9 of the 40 phones harboured Staphylococcus spp.; 3 of the 40 harboured S. aureus; 1 of the 40 harboured Micrococcus spp; 1 of the 40 grew only 1 Gram-negative bacterium; and, 1 of the 40 grew 3 Gram-negative microorganisms. The general wards had the most Gram-negative bacteria isolated ( 5 of $40,12.5 \%$ ). 


\section{Discussion}

Hospital acquired infections (HAI) are of concern to all medical practitioners, particularly infectious disease specialists and infection control staff, due to the increasing prevalence of $\mathrm{HAl}$ worldwide. As indicated previously, one possible route of transmission is through mobile phones that are contaminated..$^{3,4}$ Previous studies have shown that mobile phones carried by healthcare workers (HCW) are frequently contaminated, and are a possible source of transfer of infection from the HCW to patients as well as to inanimate objects in the wards..$^{6-8}$ However, no previous study has looked at mobile phones carried by caregivers in paediatric wards, compared contamination between different categories of HCWs or between different wards in a paediatric setting.

Our study showed that caregivers had the highest likelihood of carrying mobile phones that were contaminated, followed by medical staff with the lowest rate among the nursing staff. In the paediatric wards at $\mathrm{KEH}$, the majority of caregivers carry mobile phones. Many caregivers (mostly mothers), leave the phone on the child's bed for prolonged periods, thereby exposing the phone to possible contamination.

Medical staff particularly are likely to use their phones whilst on a ward round. The phone is used for both personal and workrelated reasons. The doctor often uses his/her phone to check doses of medication, to calculate doses of medication, to look up causes of a particular symptom or sign, to read up on side effects of drugs, etc. Not all doctors clean their hands before or after using their phones, particularly if their phone rings whilst they are examining a patient. This exposes both themselves as well as their patients to risk of transferring infections. The doctor can transfer microorganisms from the patient to their own hands, and from their hands to their phones, and from their phones to their faces, mouths and ears. In reverse, the doctor can transfer microorganisms from the phone to the patient that they are examining.

Nursing staff phones had the lowest rate of contamination. This is most likely due to the restrictions placed on the use and carrying of mobile phones by nursing staff. In King Edward hospital, nurses are not permitted to use their mobile phones during the times that they are on duty in the ward.

Surprisingly, mobile phones from both staff and caregivers from the gastroenteritis ward had the lowest rate of contamination, compared to both the neonatal unit and the general paediatric wards, where the rates were similar. This may be due to the strategic placement of posters at relevant points to reinforce the importance of handwashing in the gastroenteritis ward, or more awareness regarding infection control practices created in the gastroenteritis ward.

The most common microorganisms cultured were Staphylococcus spp., followed by S. aureus. The S. aureus isolates were all methicillin/cloxacillin susceptible. Staphylococci, in particular Staphylococcus spp., are part of the normal flora of humans. However, they are resistant to drying and may survive in the environment for months. Of the Gram-negative microorganisms, Enterobacter cloacae and Acinetobacter spp. were the most frequently cultured. These two microorganisms are the main causes of nosocomial infections, with acinetobacter being a particularly resilient microorganism that can survive in the environment for prolonged periods. Caregivers' phones were mainly contaminated by Staphylococcus spp., but were also more likely to have multiple microorganisms. This increases the risk of microorganisms being carried out of the hospital and spread into the community.

Although our sample size was small, our results indicated that one third of the mobile phones being carried and used in the paediatric wards were contaminated. This is lower than the prevalence found in other studies, but still of concern in view of the possible risk factor for spread of infection. ${ }^{10-12}$ All HCWs and caregivers need to be educated on a regular basis about infection control and the importance thereof, with emphasis on the mandatory use of disinfectants before and after any procedures and handling of phones. Standard guidelines on mobile phone usage by HCWs as well as caregivers, need to be formulated for general paediatric wards and neonatal units.

Acknowledgements - Mrs. Lee Sewnarain for administrative assistance, and for assistance with sampling. The staff and caregivers of the paediatric wards in $\mathrm{KEH}$ for their willingness to participate.

Conflict of Interest - The authors declare no conflict of interest.

Financial Support and Sponsorship - Departmental funding was used to conduct the research.

\section{References}

1. RC Pattinson. Saving babies 2010-2011: Eighth report on perinatal care in South Africa. Pretoria: Tshepesa Press, 2013.

2. World Health Organization. Prevention of hospital-acquired infections [cited 2015 Sep 17]. Available from: http://www.who.int/ csr/resources/publications/whocdscsreph200212.pdf

3. Datta P, Rani H, Chander J, et al. Bacterial contamination of mobile phones of healthcare workers. Pak J Biol Sci. 2009 July-September; 27(3):279-81.

4. Kilic IH, Ozaslan M, Karagoz ID, et al. The microbial colonisation of mobile phone use by healthcare staffs. Pak J Biol Sci. 2009;12(11):882-4.

5. Statistics South Africa: General household survey, 2013 [cited 2015 Sep 17]. Available from: http://beta2.statssa.gov.za/publications/ P0318/P03182013.pdf

6. Arora U, Devi P, Chadha A, et al. Cellphones a modern stayhouse for bacterial pathogens. Int J Clin Pract. 2009, July-September 11(3):127-9

7. Gould D. Commentary: Ulger F et al. (2009). Are we aware how contaminated our mobile phones with nosocomial pathogens? Nurs Crit Care. 2009;14(4):213-4.

8. Tambekar DH, Gulhane PB, Dahikar SG, et al. Nosocomial hazards of doctor's mobile phones in hospitals. J Med Sci (Faisalabad). 2008 January; 8(1):73-6.

9. Manual of Clinical Microbiology, 9th ed. Patrick Murray R, et al., editor Washington, DC: M Press; 2007

10. Mark $\mathrm{D}$, Leonard $\mathrm{C}$, Breen $\mathrm{H}$, et al. Mobile phones in clinical practice: Reducing the risk of bacterial contamination. Int J Clin Pract. 2014;68(9):1060-4.

11. Roy R. A surveillance study of bacterial flora associated with mobile phones in a tertiary care hospital. Int J Biomed \& Adv Res. [intranet] 2013;4(01) [cited 2015 Sep 17]. Available from: http://www.ssjournals. com/index.php/ijbar/article/view/263/261

12. Sedighi I, Alikhani M, Ramezani $S$, et al. bacterial contamination of mobile phones of healthcare providers in a teaching hospital in Hamadan Province, Iran. Arch Clin Infect Dis. [intranet] 2015;10(02):e22104 [cited 2015 Sep 17]. Available from: http:// journals.sbmu.ac.ir/infectiousinvisible/article/view/9194/7106 